
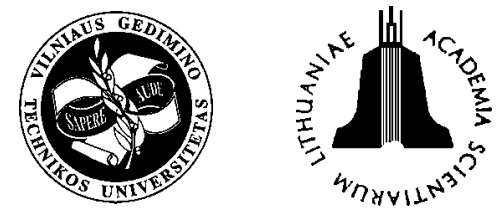

\title{
APPLICATION OF SUPERELEMENTS IN STATIC ANALYSIS OF THIN-WALLED STRUCTURES
}

\author{
Ireneusz Kreja, Tomasz Mikulski, Czeslaw Szymczak \\ Dept of Structural Mechanics, Gdansk University of Technology, Narutowicza 11/12, 80-952 Gdansk, Poland. \\ E-mails: ikreja@pg.gda.pl,tomi@pg.gda.pl,szymcze@pg.gda.pl
}

Received 16 Dec 2003; accepted 15 March 2004

\begin{abstract}
A concept of a beam superelement is suggested as a new tool in the static analysis of structures made of thinwalled members. This proposal seems to be especially attractive for treating the problems where the existing onedimensional models do not provide proper solutions. This class of problems includes, for instance, the torsion of thinwalled beams with battens and the determination of the bimoment distribution at the nodes of frames made of thinwalled members. The entire segment of the thin-walled beam with warping stiffener or the whole node of the frame is modelled with shell elements. The stiffness matrix of such thin-walled beam superelement can be estimated according to the standard procedure of the enforced unit displacements. The accuracy of the proposed one-dimensional model has proved to be comparable to that offered by the detailed FEM model where the whole structure is represented by a very large number of shell elements.
\end{abstract}

Keywords: thin-walled structures, beams, frames, stiffeners, static analysis.

\section{Introduction}

Beams and frames assembled of thin-walled members are very often used in civil engineering structures and in various machines and vehicles. The proper methods of mathematical modelling of these structures are of great importance for simulations of their behaviour. Every real structure of this type is subjected to loads that produce torsion in its members. However, due to presence of warping in beam elements, it is necessary to solve the problem of bimoment distribution in the frame nodes. In the literature and engineering practice there are various concepts of establishing the bimoments distribution [1-2], but none of them gives the general solution to this problem. The scope of this paper is limited to the beams and frames composed of thin-walled members with the bisymmetrical open cross-section. Unfortunately, the members of this kind have a small torsional stiffness and sometimes to increase it additional stiffeners like battens, transverse plates or cross trusses are necessary [3]. Similarly, as in the case of the frame node, a deformation of the member cross-section in vicinity of the stiffener location is observed.

In the present research we propose to model the structure using one-dimensional finite elements as follows:

a) in the region between nodes or stiffeners we use thin-walled elements [4] based on the classical as- sumptions of the theory of thin-walled beams of nondeformable cross-section [5];

b) in the region of nodes and stiffeners we apply superelements composed of flat shell elements. The stiffness matrix for the superelement is determined using the method of unit displacements [6] with the help of the FEM computer system MSC/NASTRAN [7], by treating the nodal zone as a complex system of plates divided into a large number of QUAD4 shell elements.

A numerical model of the analysed structure consisted of an appropriate assembly of beam elements and superelements. All calculations following the standard FEM procedures are carried out by a computer program for matrix operations, PRISM [8].

The algorithm described above has been applied to analyse several problems for beams and frames made of thin-walled members [9]. The obtained results have been positively verified with the detailed FEM model, in which the whole structure is represented as an assemblage of flat shell finite elements (MSC/NASTRAN QUAD4 shell elements). In the following two selected examples are described in detail; they are the torsion analysis of a simply supported thin-walled beam and the steel frame under transverse force. 


\section{Torsion of thin-walled beams with warping stiffeners}

\subsection{Concept of superelement}

Thin-walled beams with open cross-section can be very efficiently analysed numerically with the use of onedimensional beam model [4] based on the classical theory of thin-walled bars with non-deformable cross-section [5]. The main idea of the proposed concept is to construct an analogous one-dimensional model for a thin-walled beam segment containing warping stiffeners. The whole segment is represented as one big superelement - an assemblage of shell type finite elements QUAD4 available in the system MSC/NASTRAN (Fig 1a ${ }^{1}$ ). A proper representation of beam boundary conditions in the detailed model is obtained using the technique of rigid elements. The stiffness matrix of the 2-node beam-like element is calculated according to the common procedure of the unit enforced displacements where the resultant 14 reaction forces (shown in Fig 1b) for each enforced unit displacement form the corresponding column of the stiffness matrix.

After positive verification in the analysis of I-beams without any stiffeners $[5,9]$ the proposed procedure has been applied for I-beams with warping stiffeners.
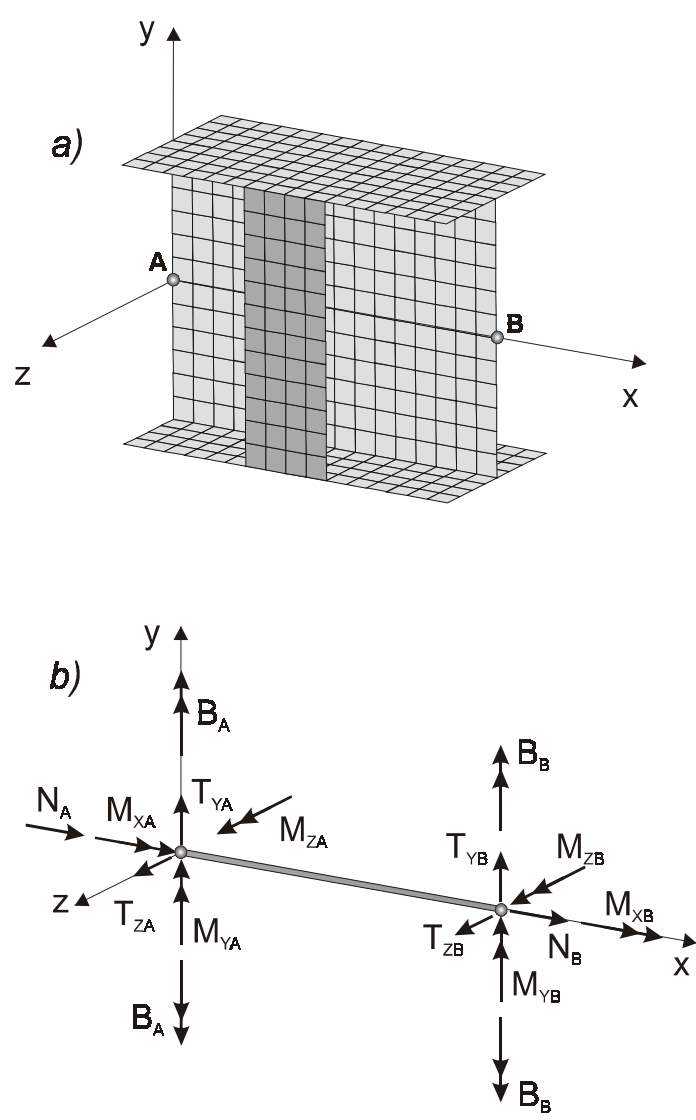

Fig 1. Thin-walled beam superelement: a) 3-D segment b) 1-D element with 14 degrees of freedom

\footnotetext{
${ }^{1}$ The FE mesh used in computations was much more dense than that shown in Fig 1.
}

\subsection{Torsion of a simply supported I-beam}

\subsubsection{I-beam without warping stiffeners}

First the I-beam without any warping stiffeners is considered. The analysed I-beam is built out of $10 \mathrm{~mm}$ thick steel panels $(E=205 \mathrm{GPa}, J=0,3)$ with the height of the web equal $30 \mathrm{~cm}$ and flanges $20 \mathrm{~cm}$ wide. A simply supported beam is subjected to the torque of $1 \mathrm{kNm}$ as shown in Fig 2.

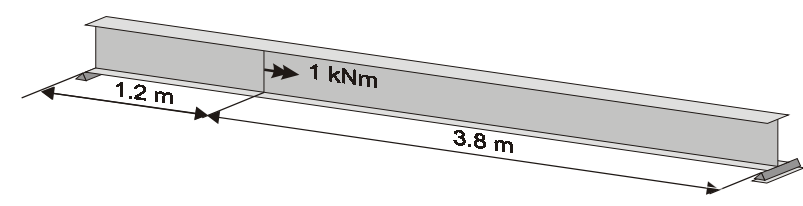

Fig 2. Simply supported I-beam undergoing torsion

The analytical one-dimensional model of the I-beam consists of seven standard I-beam elements [4]. Due to the loading conditions the number of degrees of freedom in the present analysis can be reduced to 2 DOFs per each nodal point. The second one-dimensional model has been constructed of seven superelements $(3 \times 0,6 \mathrm{~m}$ $+4 \times 0,8 \mathrm{~m})$. All calculations for both one-dimensional models have been carried out with the computer program for matrix operations, PRISM [8]. To provide a reference solution for the analysed problem a detailed FEM model has been applied where the whole beam has been represented as the assemblage of over twenty two thousand QUAD4 shell elements of the system MSC/ NASTRAN. The graphs of the torsion angle, bimoment and the warping distribution obtained with both those models are given in Fig 3, 4 and 5, respectively.

In all graphs presented in Figs 3 to 5 a very good agreement can be observed between the results of the analytical model and those obtained with superelements. Solutions from both the one-dimensional models have been confirmed additionally by the detailed FEM model. The positive response achieved in this comparative test forms a good foundation for the applying the concept of

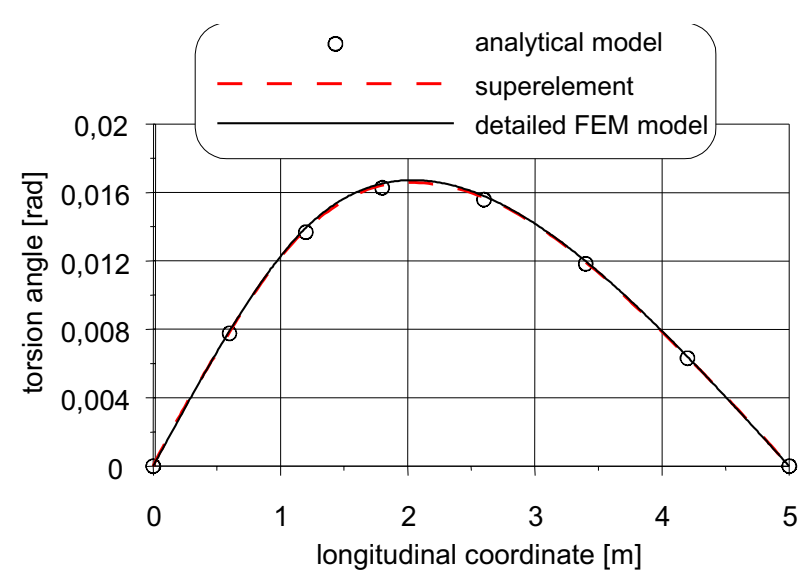

Fig 3. Torsion angle for I-beam without stiffeners 
the superelement in the analysis of the I-beam with different warping stiffeners added at the loaded cross-section. In the following calculations the superelements are used to model only the thin-walled beam segments containing stiffeners, assuming that the remaining part of the thin-walled beam can be effectively represented by the one-dimensional beam element [4].

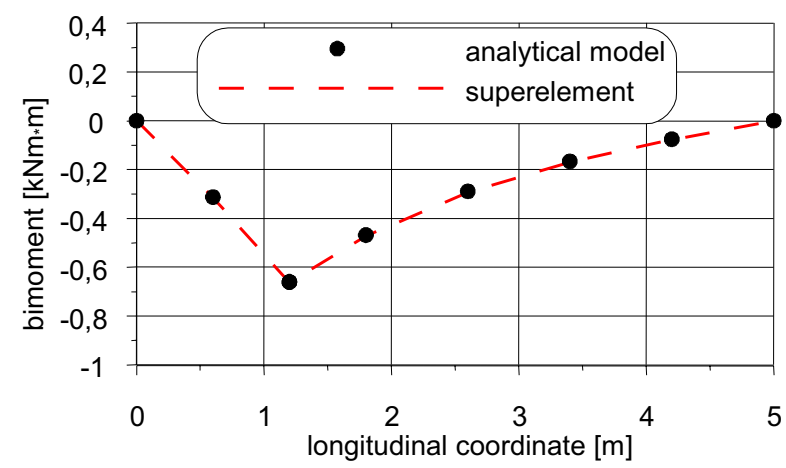

Fig 4. Bimoment distribution for I-beam without stiffeners

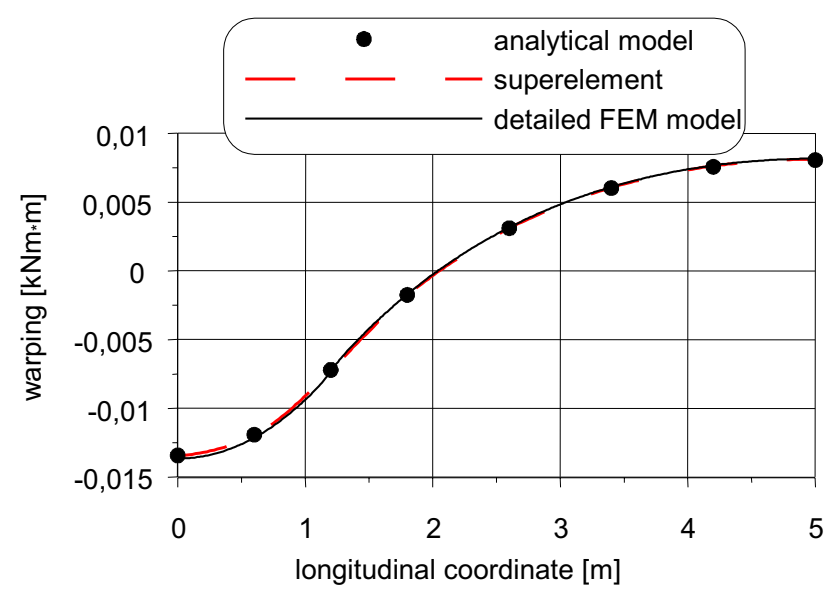

Fig 5. Warping for I-beam without warping stiffeners

\subsubsection{I-beam with lateral diaphragms}

The first type of the warping stiffener considered here are lateral diaphragms located in the cross-section at $\mathrm{x}=1,2 \mathrm{~m}$ where the torque is applied. The lateral diaphragms are constructed as two steel panels $10 \mathrm{~mm}$ thick located on both sides of the web of the I-beam. The analysed simply supported I-beam with lateral diaphragm is shown in Fig 6.

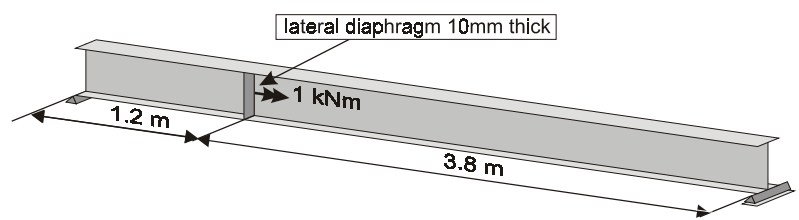

Fig 6. A simply supported I-beam with lateral diaphragm
The analytical, one-dimensional model of the I-beam with the lateral diaphragm consists of seven standard I-beam elements [4] accompanied by the warping spring with the stiffness estimated according to the formula:

$$
k_{B}=\frac{G t^{3} b h}{3},
$$

where $t$ is the thickness of the diaphragm, $b$ stands for the its width and $h$ for the height with $G$ being the shear modulus.

The second one-dimensional model of the analysed beam is constructed of five standard I-beam elements [4] and one superelement $40 \mathrm{~cm}$ long. Here again the detailed FEM model is applied to provide an additional reference solution.

The results obtained with those three models are illustrated by the graphs of the torsion angle, the bimoment and the warping given in Figs 7 to 9 .

Examining the presented graphs one can notice a very good agreement of the results obtained with the three models used in the calculations. The analytical formula given in (1) seems to be a good estimation of the warping spring stiffness for the lateral diaphragms. On the other hand, comparing the graphs given in Fig 3 and in Fig 7, someone can notice that the torsion stiffness of the I-beam does not increase significantly after the lateral diaphragm is appended.

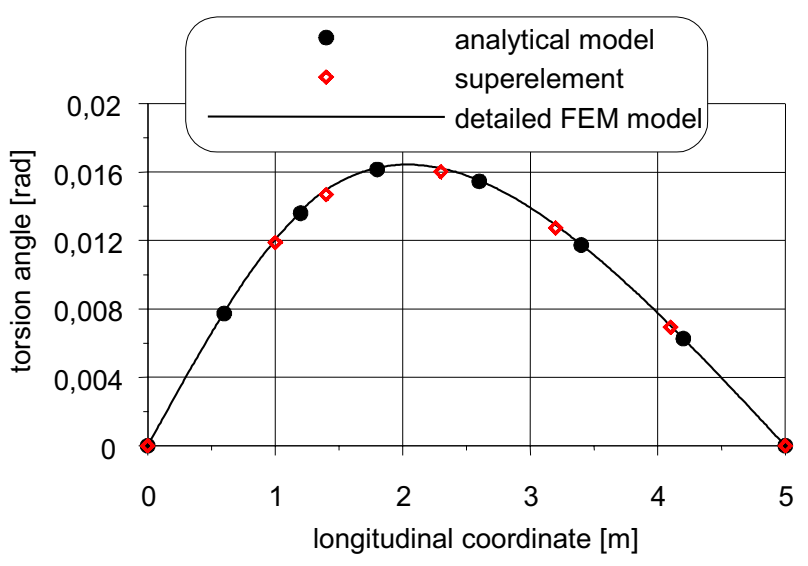

Fig 7. Torsion angle for I-beam with lateral diaphragm

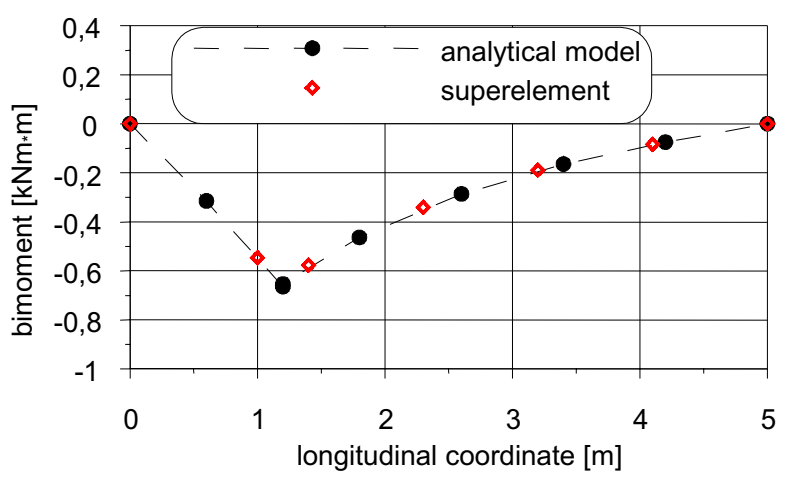

Fig 8. Bimoment for I-beam with lateral diaphragm 


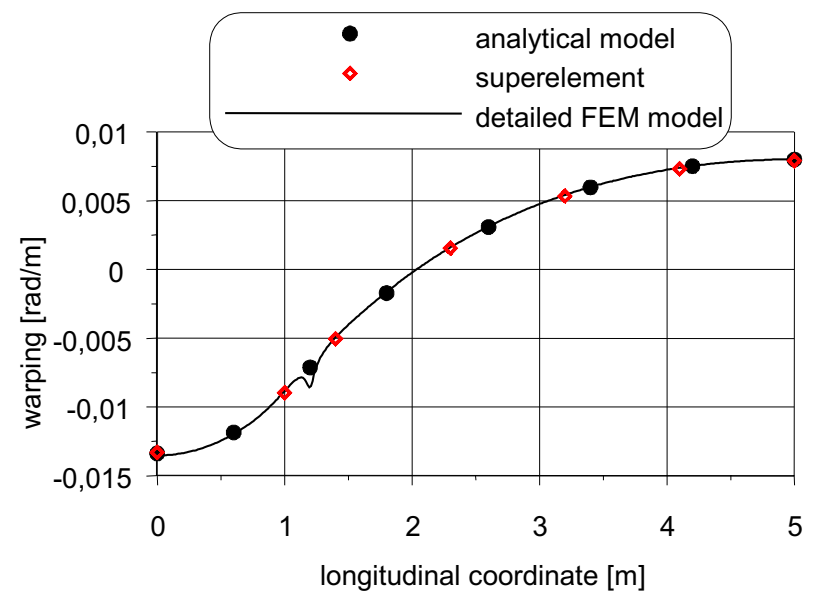

Fig 9. Warping for I-beam with lateral diaphragm

The value of the warping in the detailed FEM model has been calculated as the derivative of the torsion angle taken with the respect to the longitudinal coordinate. A numerical evaluation of the derivative has been performed by the central difference method. A visible disturbance can be observed in the distribution of the derivative of the torsion angle within the region of the diaphragms. Both one-dimensional models give more regular solutions.

\subsubsection{I-beam with battens}

In the second example of the I-beam with the warping stiffeners, the battens have been added in the crosssection loaded with the torque. The battens are constructed as two steel panels parallel to the web of the I-beam and located on both its sides. The thickness of battens is equal to $10 \mathrm{~mm}$. The analysed I-beam with battens is shown in Fig 10.

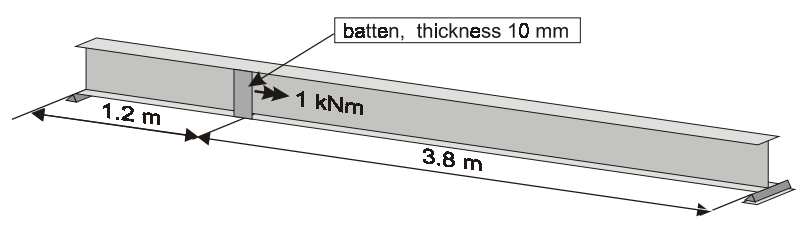

Fig. 10. Simply supported I-beam with battens

The same three models as described in 2.2.2 have been applied in the current analysis with the only difference that the stiffness of the warping spring representing the battens is estimated according to the formula

$$
k_{B}=\frac{E b_{b}^{3} b_{1}^{2} t}{2 h} \frac{1}{1+1.2 \frac{E}{G}\left(\frac{b_{b}}{h}\right)^{2}}
$$

with $h, t$ and $b_{b}$ standing for the height, the thickness and the width of the batten, respectively, $E$ being the Young modulus, and $b_{1}$ representing the distance between the battens in the beam cross-section.
The graphs of the torsion angle, the bimoment and the warping obtained for the I-beam with the battens are presented in the Figs 11 to 13, respectively.

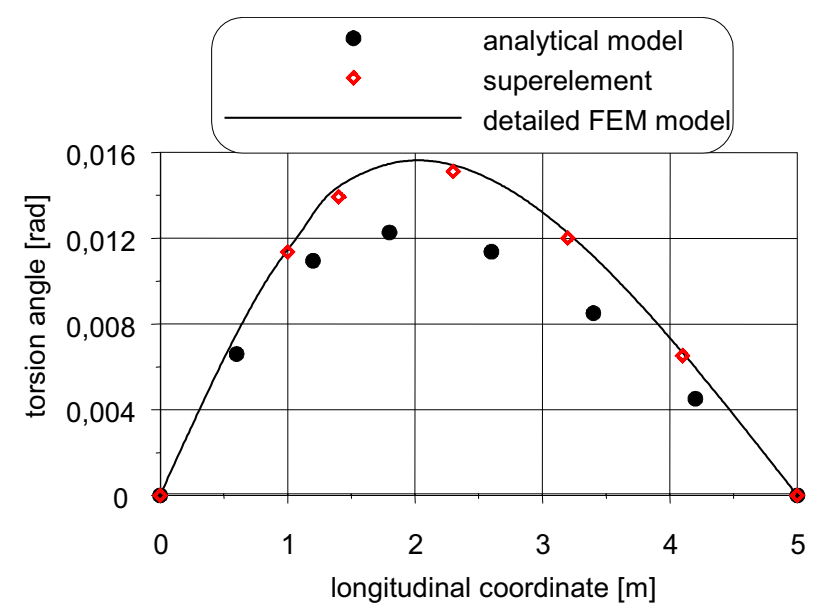

Fig 11. Torsion angle for I-beam with battens

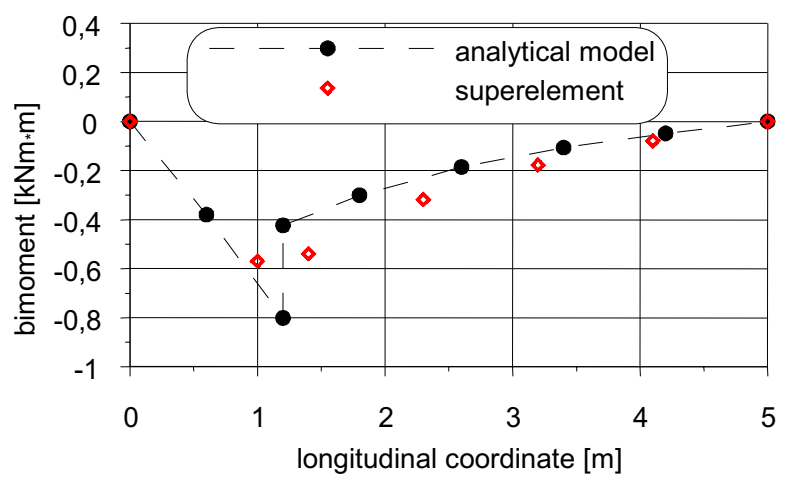

Fig 12. Bimoment for I-beam with battens

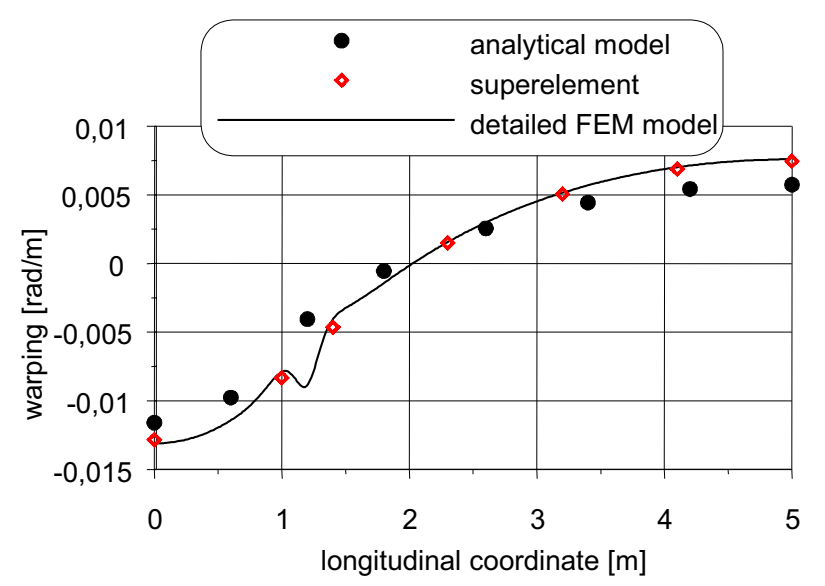

Fig 13. Warping for I-beam with battens

The graphs presented in Fig 10 show that the application of the superelement allows for a very accurate prediction of the torsion angle of the beam as compared with the results obtained with the detailed FEM model. On the other hand, the analytical model utilising the 
warping spring is noticeably stiffer. Since the analytical model performed quite well for the I-beam without battens, it is obvious that the spring stiffness is a critical issue of that approach. Relatively big jump in the graph of the bimoment (Fig 12) obtained from the analytical model confirms an opinion that the stiffness of the warping spring is overestimated.

\subsubsection{I-beam with box stiffeners}

The last warping stiffener considered in this example is constructed as a closed box made of steel panels 10 mm thick. The analysed simply supported I-beam with box stiffeners is presented in Fig 14.

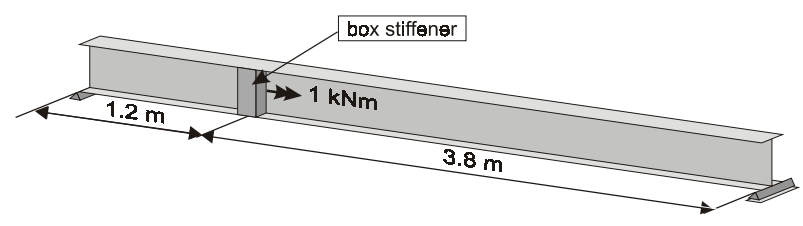

Fig 14. Simply supported I-beam with box stiffeners

In the literature one can find the following suitable formula for the stiffness of the warping spring representing the box stiffener

$$
k_{B}=\frac{2 G b_{b} t b h}{b+b_{b}}
$$

with $b_{b}$ standing for the width of the panel parallel to the web.

The same 3 models, as in the previous examples, have been applied here to obtain the graphs of the torsion angle, the bimoment and the warping for the I-beam with the box stiffeners presented in the Figs 15 to 17 , respectively.

Looking at the graph of torsion angle presented in Fig 15 one can notice that the box stiffener provides much larger increase of the torsional stiffness of the I-beam than any one of the stiffeners considered before.

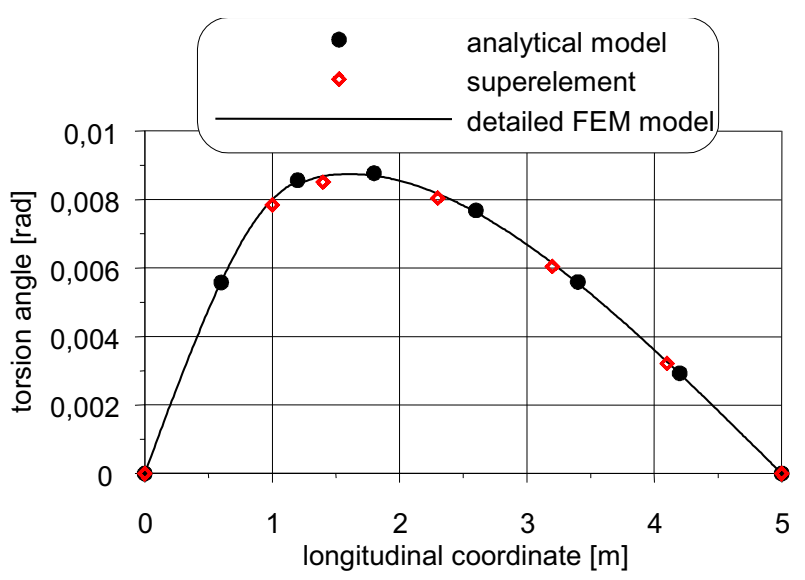

Fig 15. Torsion angle for I-beam with box stiffeners

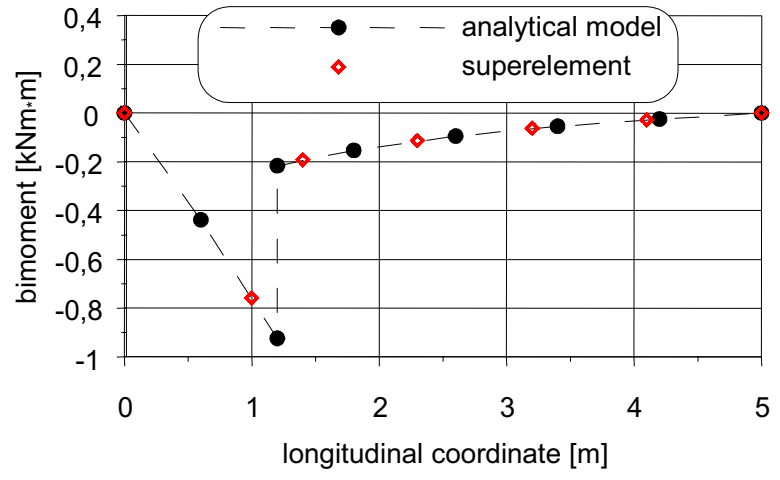

Fig 16. Bimoment for I-beam box stiffeners

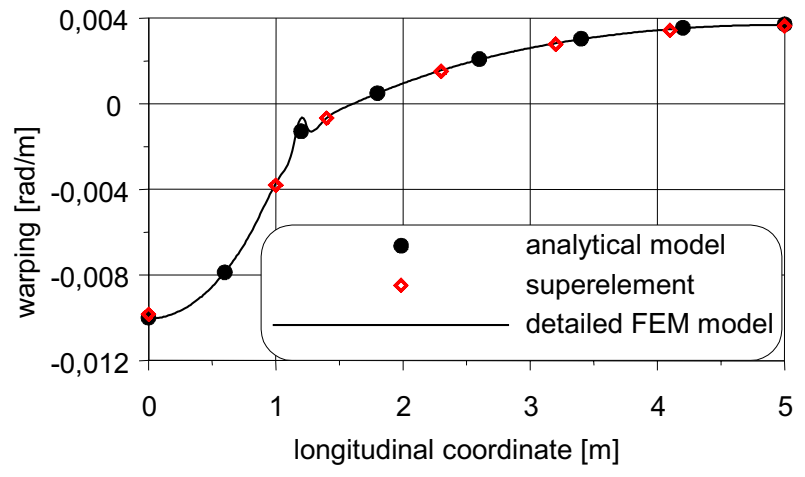

Fig 17. Warping for I-beam with box stiffeners

Since all three models applied in the analysis give similar responses it seems that the stiffness of the warping spring representing the box stiffener can be correctly established by using the formula (3). An additional support for that conclusion can be observed in Fig 16 where the bimoment distribution obtained by the analytical model is in an excellent agreement with the results of the calculations performed with the superelement approach.

Summarising the conclusions for the presented example, it can be stated that the analytical model utilizing the concept of the warping spring gives a correct solution for the pure torsion analysis of the thin-walled beam with lateral diaphragms or with box stiffeners. However, this approach does not provide a right response for the case of the battens. On the other hand, a correct solution can be obtained for any one of the considered stiffeners when the concept of the superelements is applied.

It should be emphasised that the effect of the warping stiffeners depends very much on their location. One can imagine that for the analysed beam the application of warping stiffeners at the supports would significantly increase this effect.

\section{Thin-walled frames}

Thin-walled frames are common steel constructions in various civil engineering objects, like industrial or shopping halls, island station roofs or high buildings. 
Such constructions consist of many beams, columns, lateral or wind bracings connected in nodes. Warping of the member cross-section plays a significant role in stress and deformation distribution. It depends on the frame node construction or the stiffeners located along the beam span.

Presented considerations are restricted to the plane frames. The superelement technique is applied in the modelling of the warping effects in frame nodes and thinwalled bar elements with stiffeners, where the non-deformable cross-section assumption of the classic thinwalled theory is not fulfilled.

\subsection{Node superelement}

Nodes of thin-walled frames are the most significant elements, where the thin-walled beams are connected at different angles. In this case, within one dimensional classic beam theory, it is impossible to determine the warping distribution and bimoments as internal forces. There are no equilibrium conditions of bimoments in nodes and occurrence of significant deformations of cross section is noted, which is in inconsistency with the assumptions of the thin-walled beam theory - non-deformable cross-section. Also the node construction plays an important role in the warping distribution phenomena. The node superelement is thus a suitable model to take all the effects mentioned into account.

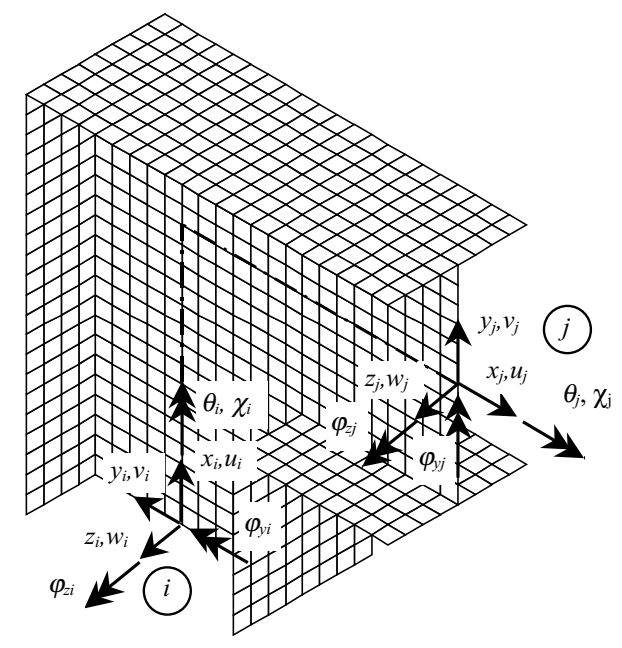

Fig 18. Node superelement, 14 degrees of freedom

he stiffness matrix of the thin-walled node superelement has been calculated according to the common procedure of the unit enforced displacements where the resultant 14 reaction forces (shown in Fig 18) for each enforced unit displacement form the corresponding column of the stiffness matrix. Herein the axial stretching, bending and shearing behaviour of the thin-walled superelement node is coupled with torsional performance.

MSC/NASTRAN for Windows [7] FEM system containing QUAD4 shell elements has been employed in the study of the detailed node superelement model. Four- teen unit unforced stages have been analysed to obtain 144 stiffness matrix coefficients. Many of them are approached to zero - depending on the nodal force coupling. The 4-node shell elements QUAD4 available in that system have been used to model the entire segment of the thin-walled node.

\subsection{Comparative analysis for two-member frame}

Let us consider a simple two member frame built of I-beams shown schematically in Fig 19. The frame is subjected to the torque $M=1 \mathrm{kNm}$ acting at the midspan of the horizontal beam. There are not stiffeners in the frame.

The numerical static analysis of the frame is carried out using three different models:

1) classic beam theory model, where the frame is modelled by standard beam elements with 12 degrees of freedom (6 DOFs per each node), the warping effect is neglected;

2) thin-walled beam model [4] based on the classical theory of thin-walled bars with non-deformable cross-section [5] combined with the node superelement described in 3.1. Both element types have 14 degrees of freedom and take into account the warping effects.

3) detailed FEM model, where the whole frame is modelled as an assembly of QUAD4 shell elements (Fig 20) available in MSC/NASTRAN. The four-node QUAD4 shell elements have 24 degrees of freedom.

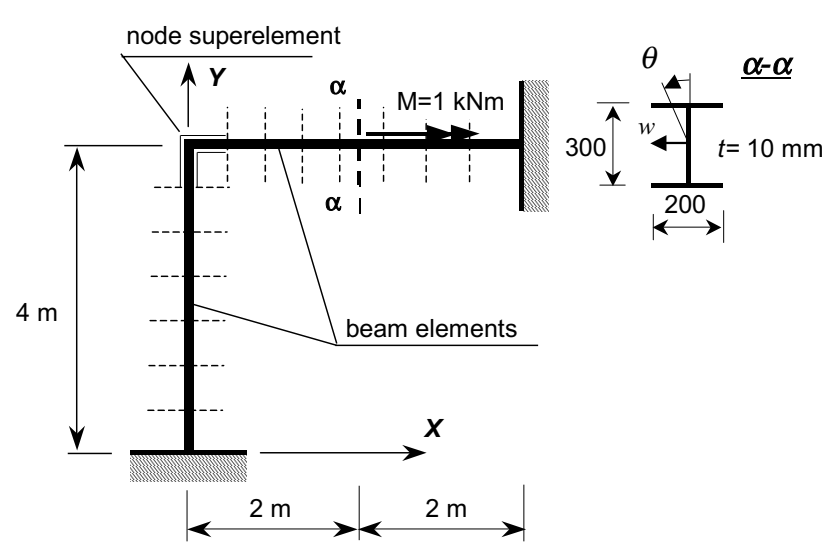

Fig 19. Frame modelled by thin-walled elements with node superelement

In Figs 21 and 22 the distribution of torsion angle along the column and along the horizontal beam obtained with the aid of the models under consideration is shown. The solution of the three-dimensional frame model discretised by shell elements is assumed to be more adequate to the real construction and has been treated as a reference model. The classic beam theory model gives a solution quite different from those of the shell model. The model consisting of the thin-walled beam elements 
together with the node superelement provides a solution very close to the comparative shell model. A serious discrepancy between the classic beam theory solution and the results corresponding to more accurate models is observed, especially in the horizontal member.

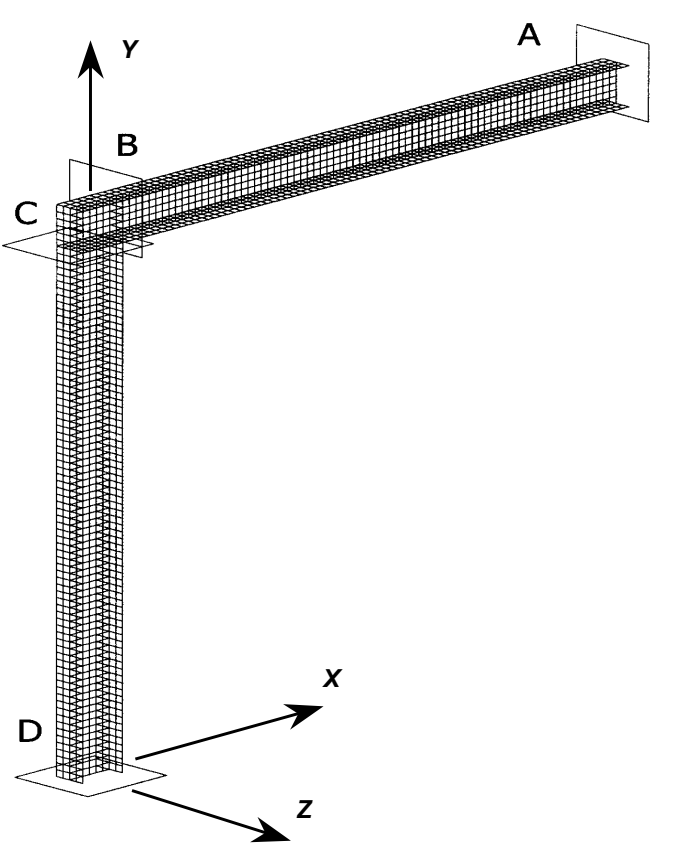

Fig 20. Detailed FEM model, QUAD4 shell elements of MSC/NASTRAN

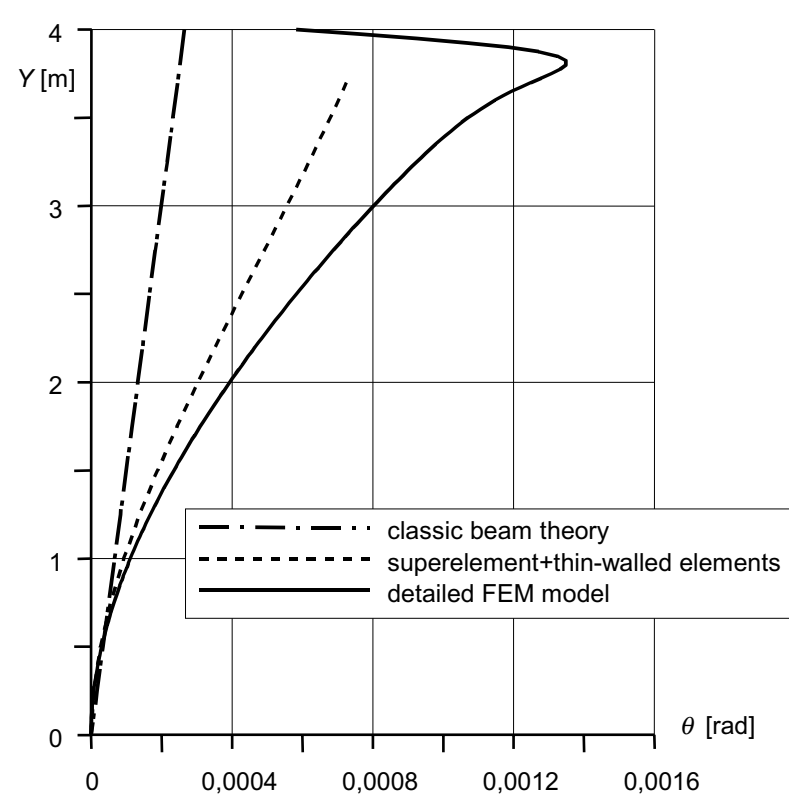

Fig 21. Distribution of torsion angle $\theta$ along the frame column

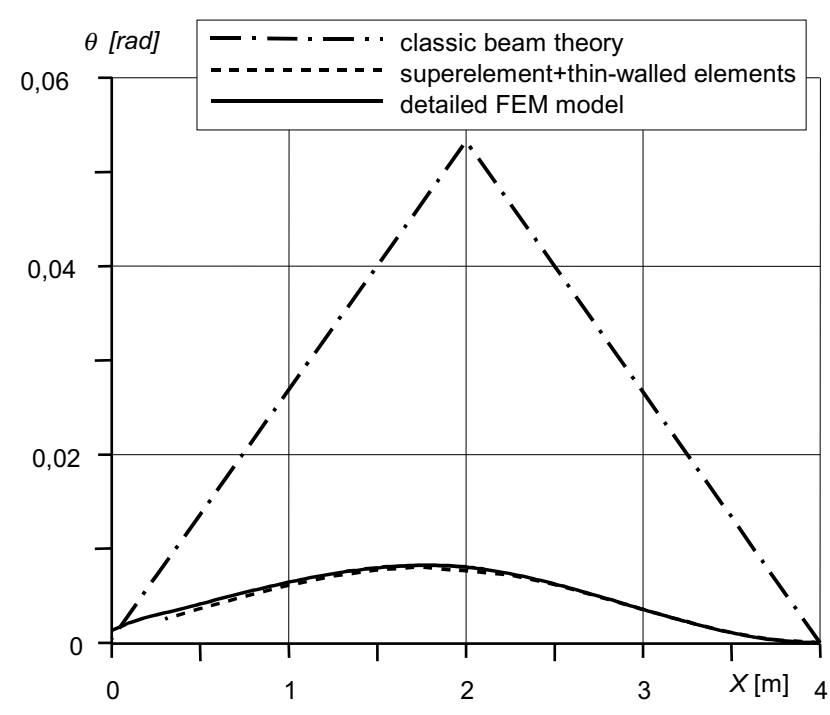

Fig 22. Distribution of torsion angle $\theta$ along the horizontal beam

\subsection{Plane frame with battens}

Warping stiffeners are very often used in thin-walled frames to increase the frame stiffness for torsion. Let us consider a plane frame with three double battens placed along the horizontal beam as presented in Fig 23.

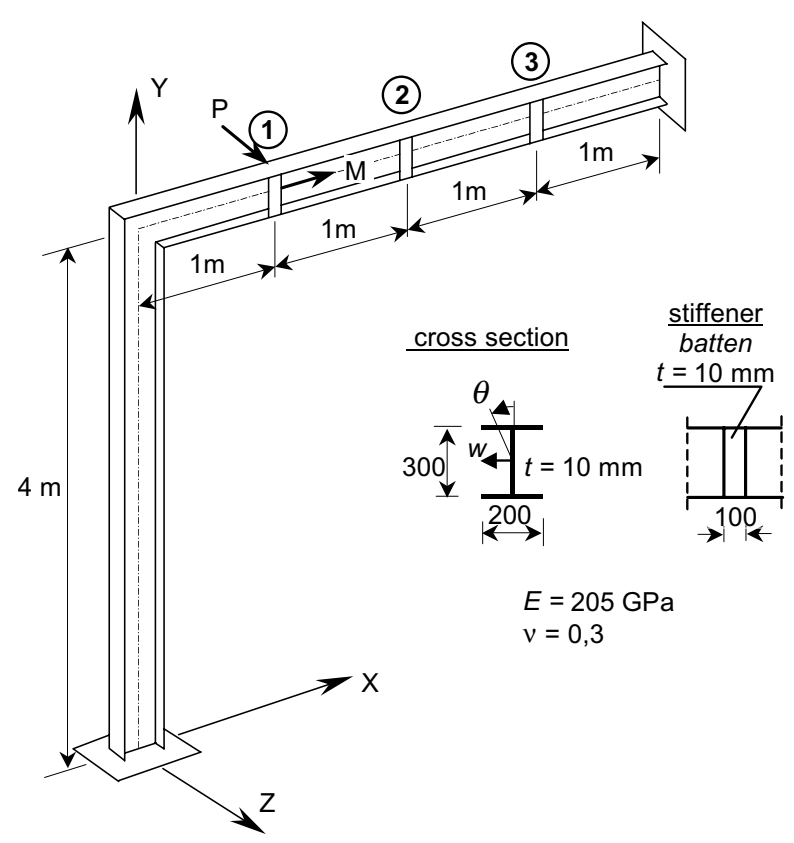

Fig 23. Thin-walled frame with battens

The node and the batten superelements have been implemented using a one-dimensional model shown in Fig 24. Each of the FEM elements used in this model has 14 degrees of freedom: three translations, three rotations and warping at each element node. 


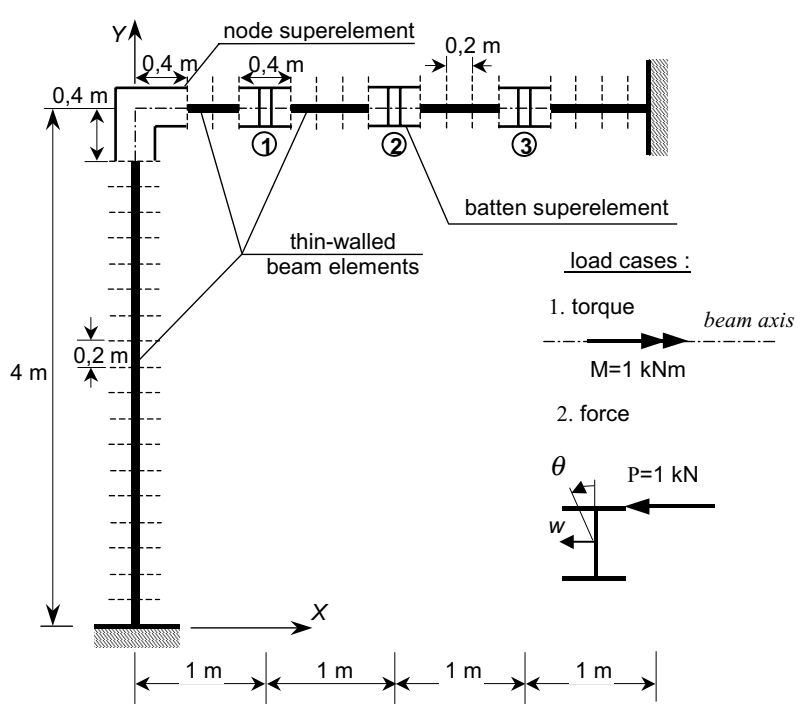

Fig 24. One-dimensional FEM model, frame with node and batten superelements
The results one-dimensional frame model is compared with a detailed FEM model (MSC/NASTRAN, QUAD4 shell elements) where the non-deformable cross section condition is not enforced.

Two load cases are considered:

1) torque $\mathrm{M}=1 \mathrm{kNm}$ applied sequentially at points 1 , 2 and 3 ;

2) transverse horizontal force $P=1 \mathrm{kN}$ applied to the upper flange at the point 1 .

Both load cases are graphically presented in Figs 23 and 24.

The distribution of the torsion angle $\theta$ along the horizontal beam for load case 1 is presented in Figs 25 to 27 . The influence of battens responsible for increasing the frame beam torsion stiffness is visualised by reducing the torsion angle $\theta$. The static analysis solutions of the one-dimension frame model with superelements are very close to the results obtained for the shell model.

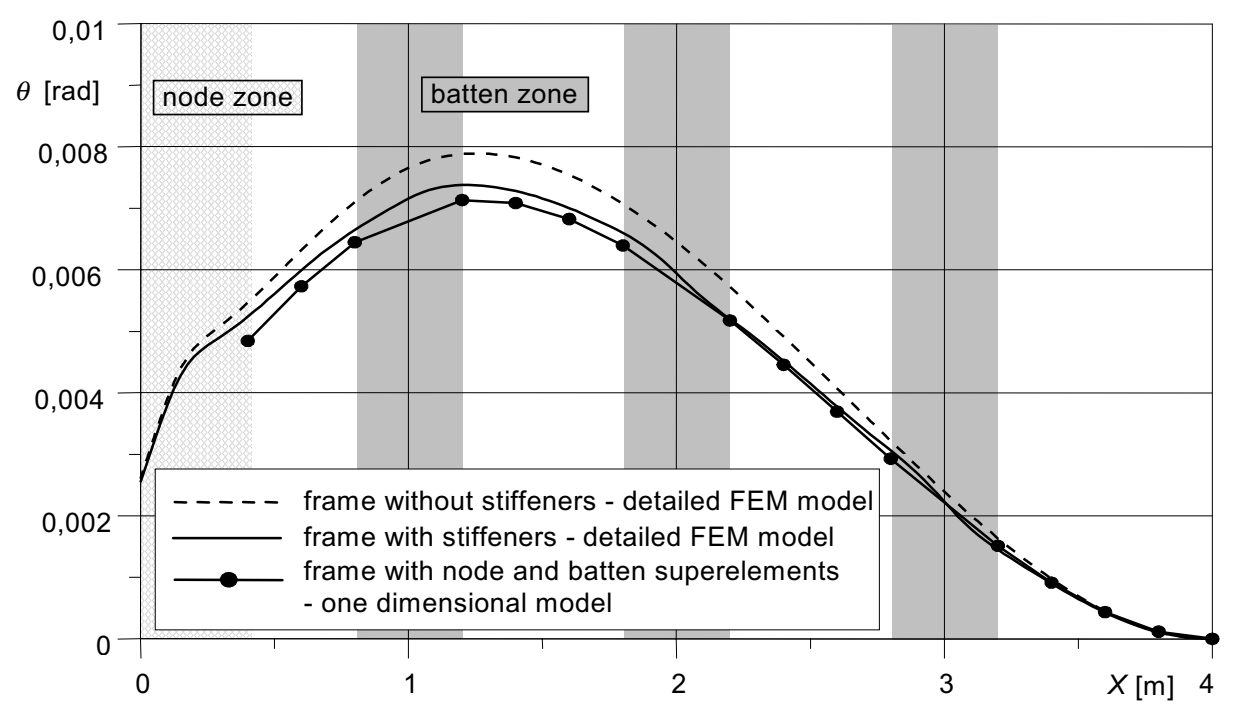

Fig 25. Torsion angle $\theta$ distribution along the horizontal beam, load case 1 , unit torque $M=1 \mathrm{kNm}$ acting at point 1

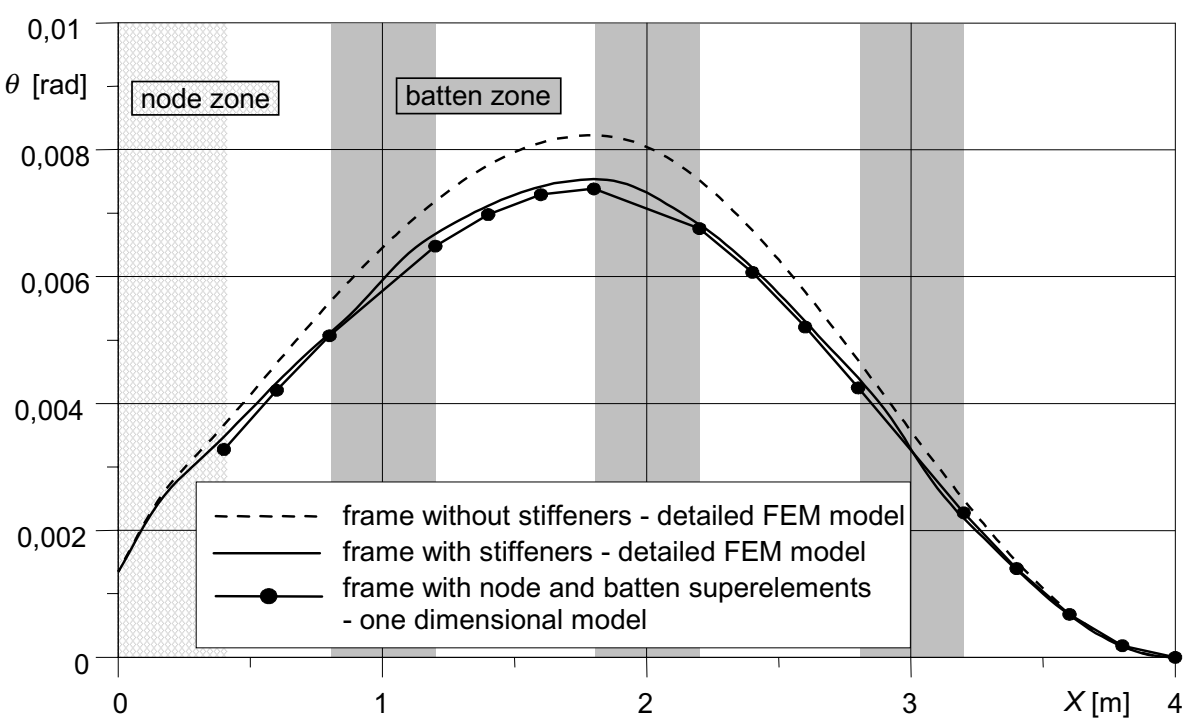

Fig 26. Torsion angle $\theta$ distribution along the horizontal beam, load case 1 , unit torque $M=1 \mathrm{kNm}$ acting on point 2 
The results for the second load case (transverse horizontal force $P=1 \mathrm{kN}$ applied at point 1 ) are presented in the Figs 28 and 29. Here, again, a quite good agreement can be observed between the results of the onedimensional model with superelements and the detailed FEM model utilising shell elements.

\section{Conclusions}

The one-dimensional thin-walled beam 2-node element proposed by Barsoum \& Gallagher [4] proved to be an efficient tool for the numerical analysis of thinwalled beams. The formulation of this element is based on the classical theory of thin-walled bars with non-deformable cross-section [5]. The warping of the thin-walled beam is included as the seventh degree of freedom at each nodal point, whereas the remaining six DOFs are usual 3 translations and 3 rotations common for the most space frame formulations. The results calculated with this element are in very good agreement with the reference solutions obtained by means of the detailed FE model where the thin-walled beams were modelled as assemblages of shell elements.

One should notice that the classical theory of thinwalled bars with non-deformable cross-section [5] is not appropriate for modelling the behaviour of the thin-walled beams with warping stiffeners, especially with battens. Due to the specific formulation of the warping DOF, which is defined in the local coordinate system of the beam, considerable problems appear also when the thinwalled beam elements are applied in the analysis of frames built out of thin-walled members. In both cases the one-dimensional model of the thin-walled beam can be effectively upgraded by employing the concept of superelements proposed by the authors of the paper.

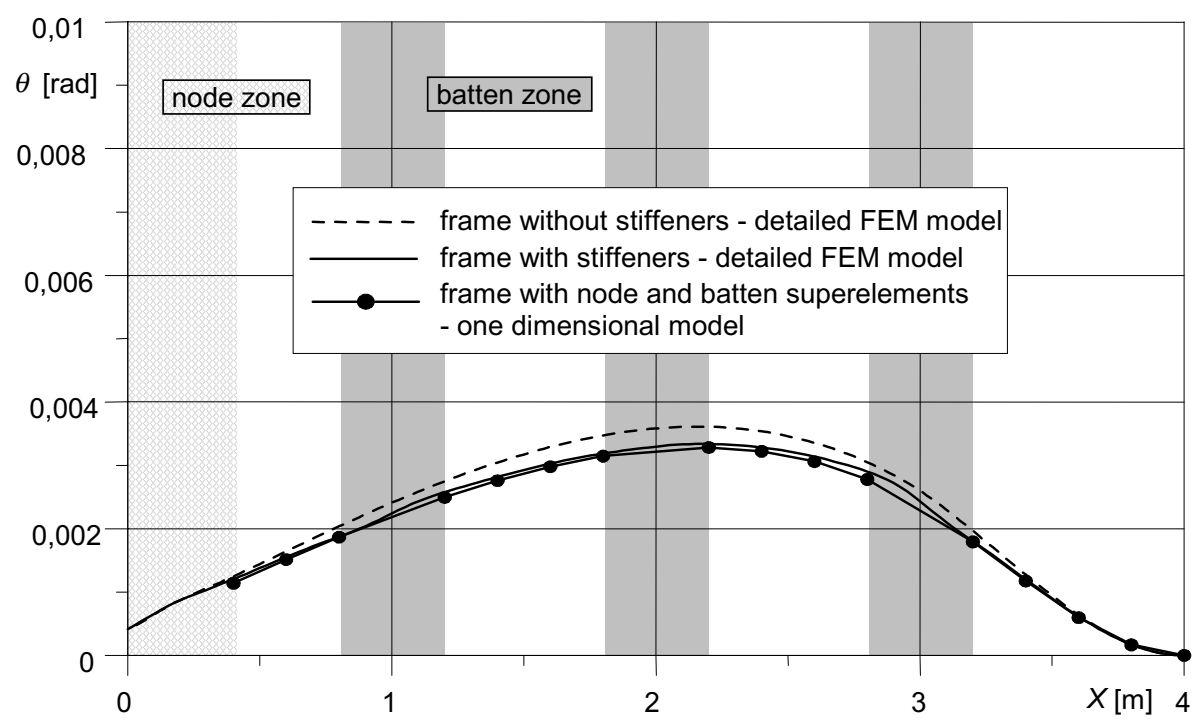

Fig 27. Torsion angle $\theta$ distribution along the horizontal beam, load case 1 , unit torque $M=1 \mathrm{kNm}$ acting on point 3

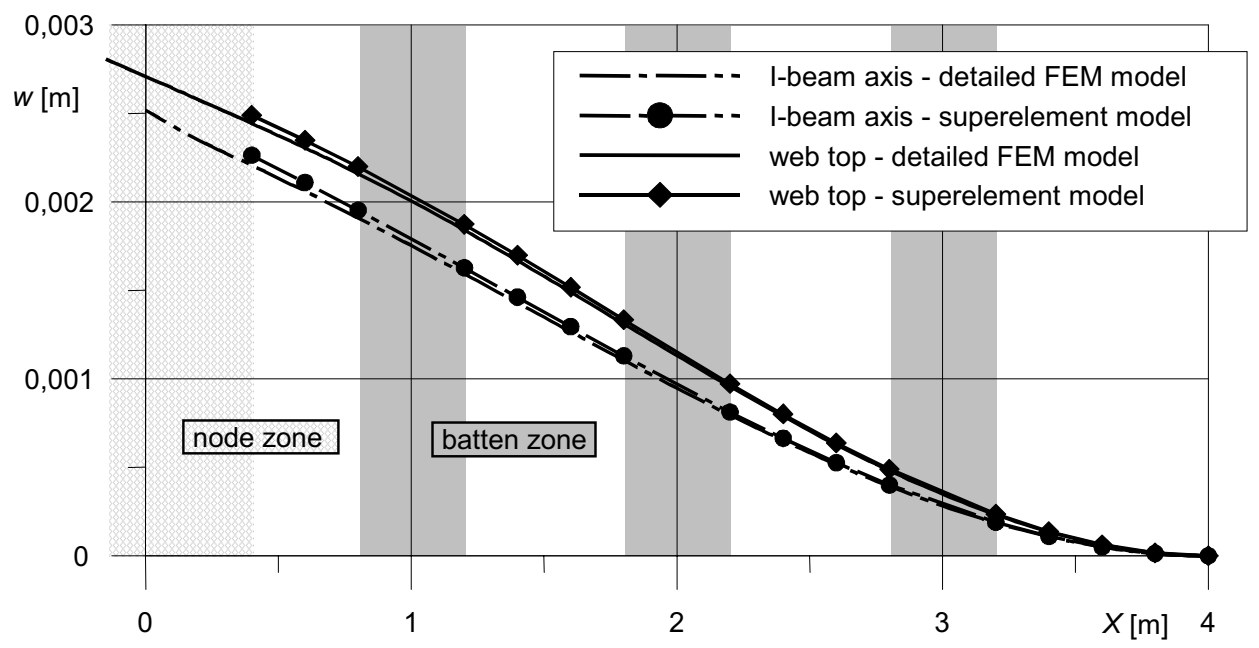

Fig 28. Distribution of displacement $w$ along the horizontal beam, unit force $P=1 \mathrm{kN}$ perpendicular to the frame plane applied at point 1 of the web and the top flange joint 


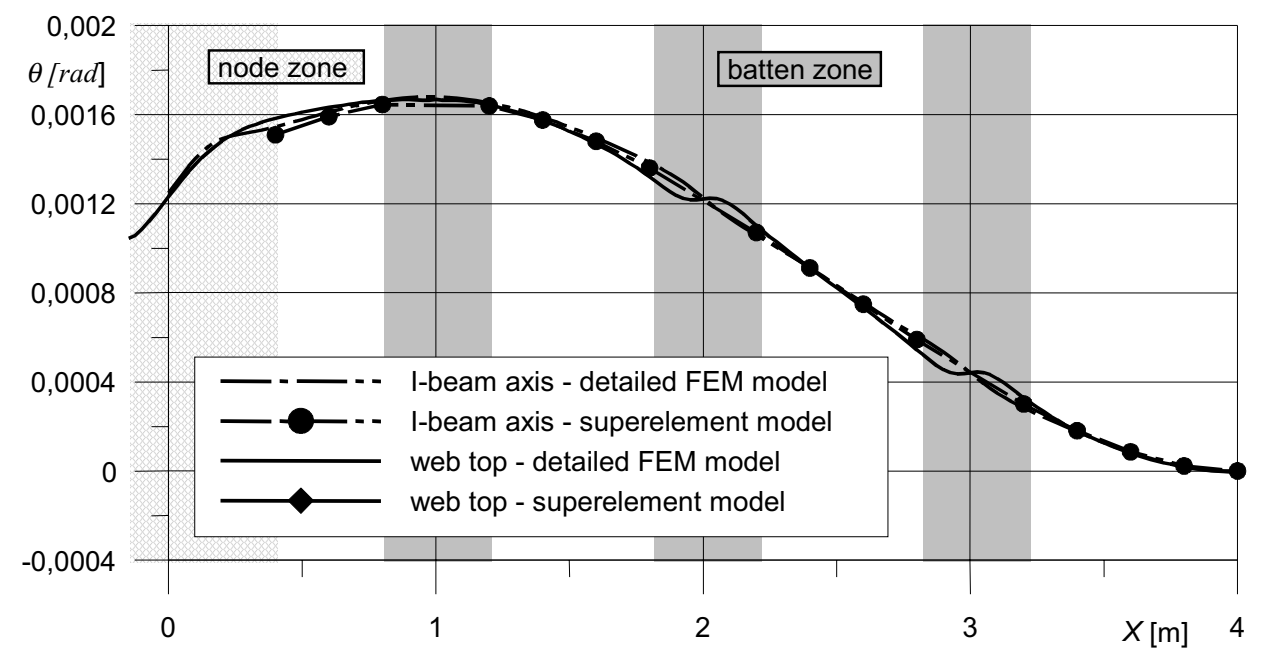

Fig 29. Distribution of torsion angle $\theta$ along the horizontal beam, unit force $P=1 \mathrm{kN}$ perpendicular to the frame plane applied at point 1 of the web and the top flange joint

The presented numerical examples have shown that the one-dimensional beam and frame models utilising the concept of the superelement for the segments containing warping stiffeners and for frame nodes offers the accuracy which is comparable to that of the detailed FEM model, with an additional remark that the numerical size of the former is just a fraction of the size of the latter.

Although the superelement offers an equivalent precision in the thin-walled beams analysis as the mentioned above element of Barsoum \& Gallagher [4], they are not recommended in the analysis of straight thin-walled beams without any stiffeners - one should remember that the stiffness matrix of the B\&S thin-walled beam element is given in explicit form [4] whereas a rather complex FEM analysis is required to establish the stiffness matrix of the superelement.

The positive results of the numerical studies carried out by the authors proved that frames constructed of thinwalled members can be very effectively analysed with the combined one-dimensional model where the superelements are applied only to model frame nodes or beam segments containing stiffeners with the remaining part of the structure represented by the one-dimensional beam element [4]. The approach recommended in the present paper has been successfully applied also in the sensitivity analysis of thin-walled structures $[9,10]$.

\section{Acknowledgements}

The financial support of the State Committee for Scientific Research (KBN, Poland) under Grant No 7 T07E 01519 is gratefully acknowledged.

\section{References}

1. Szmidt, K. Analysis of frames made of thin-walled members. Engng. Trans., 23, 1975, p. 447-442 (in Polish).

2. Yang, Y-B.; McGuire, W. A procedure for analysing space frames with partial warping restraints. Int. J. Numer. Meth. Engng., 20, 1984, p. 1377-1398.

3. Szewczak, R. M.; Smith, E. A.; DeWolf, J. T. Beams with torsional stiffeners. J. Struct. Engng. ASCE, 109, 1983, p. 1635-1647.G.

4. Borsoum, R.; Gallagher, R. H. Finite element analysis of torsional and torsional-flexural stability problems. Int. J. Numer. Methods Engng., 2, 1970, p. 335-352.

5. Vlasov, V. Z. Thin-Walled Elastic Beams, Israel Program for Scientific Translations, Jerusalem 1961; Oldbourne Press, London, 1961.

6. Kreja, I.; Szymczak, C. On numerical analysis of a thinwalled I-beam with warping stiffeners under torsional loads, CMM-2003 - Computer Methods in Mechanics, June 3-6, 2003, Gliwice, Poland.

7. MSC/NASTRAN for Windows, Installation and Application Manual, MacNeal-Schwendler Corporation, 1995.

8. Branicki, C. J.; Wekezer, J. W. Symbolic matrix interpretive system for teaching Structural Analysis, Int. J. Applied Engineering Education, 6, 1990, p. 451-460.

9. Szymczak, C.; Kreja, I.; Mikulski, T.; Kujawa, M. Sensitivity Analysis of Beams and Frames Made of Thin-Walled Members, Gdansk University of Technology Publishers, Gdansk, 2003.

10. Kreja, I.; Mikulski, T.; Szymczak, C. Sensitivity analysis of thin-walled beams and frames. In: The $8^{\text {th }}$ International Conference "Modern Building Materials, Structures and Techniques". Vilnius, 2004. 